\title{
Spatial coherence of an optical turbulent beam in a biased photorefractive crystal due to the spatiotemporal modulation instability
}

\author{
Fang-Wen Sheu ${ }^{\mathrm{a}, *}$, Ming-Feng Shih ${ }^{\mathrm{b}}$ \\ a Department of Applied Physics, National Chiayi University, Chiayi 60004, Taiwan \\ b Department of Physics, National Taiwan University, Taipei 10617, Taiwan
}

Received 14 August 2006; received in revised form 25 May 2007; accepted 2 June 2007

\begin{abstract}
We report the experimental observation of the dynamic pattern formation of a broad coherent light beam in a biased photorefractive crystal due to the spatiotemporal modulation instability. When the nonlinearity exceeds a specific threshold, the coherent light beam not only breaks up into light spots due to the modulation instability but also fast fluctuates both spatially and temporally, forming an optical turbulent beam, which behaves as a quasi-homogeneous speckled beam or a partially incoherent beam. We investigate the spatial coherence property of an optical turbulent beam from the visibility of the averaged double-slit interference fringe. We also numerically demonstrate the visibility variation of the instantaneous interference fringe of an optical turbulent beam.
\end{abstract}

(c) 2007 Elsevier B.V. All rights reserved.

PACS: $42.25 . \mathrm{Hz} ; 42.25 . \mathrm{Kb} ; 42.65 . \mathrm{Jx} ; 42.65 . \mathrm{Sf} ; 42.60 . \mathrm{Jf}$

Keywords: Interference; Coherence; Self-focusing; Optical instabilities; Pattern formation

\section{Introduction}

Several important reports of pattern formation due to the modulation instability in spatially incoherent light beams [1-3], have attracted again many research interests in photorefractive nonlinear optics since 2000. Modulation instability is a nonlinear process, in which small amplitude and phase perturbations (due to the noise) grow rapidly under the combined effects of the nonlinear self-focusing effect and the light diffraction. Thus, as illustrated in Fig. 1, a broad optical beam has a tendency to disintegrate into filaments or break up into light spots during propagation. In the report of the incoherent modulation instability [1], the nonlinearity threshold for pattern formation to occur spontaneously from noise was found to depend on the coherence property of the incoherent beam. The fila-

\footnotetext{
${ }^{*}$ Corresponding author. Tel.: +886 5 2717993; fax: +886 52717909.

E-mail address: fwsheu@mail.ncyu.edu.tw (F.-W. Sheu).
}

ments that emerge from the incoherent modulation instability process are actually trains of almost ideal incoherent solitons $[4,5]$. The key nature for the incoherent solitons to occur is that the nonlinear material within which the light propagates must be "noninstantaneous", i.e., the material gives rise to the nonlinear refractive index basing on the time-averaged intensity profile and not on the instantaneous rapidly varying speckled intensity pattern. In other words, the material is indifferent to any rapid local intensity fluctuation that is faster than the material can respond. Therefore, given that the intensity variation of the light beam is much faster than the charge re-distribution process, the photorefractive screening nonlinearity is of course noninstantaneous, allowing the spatially and temporally incoherent solitons to occur [6].

In order to investigate how a partially incoherent soliton reacts in the medium that somewhat can respond to the spatially fluctuating intensity of the soliton beam, we have studied experimentally and numerically with the 


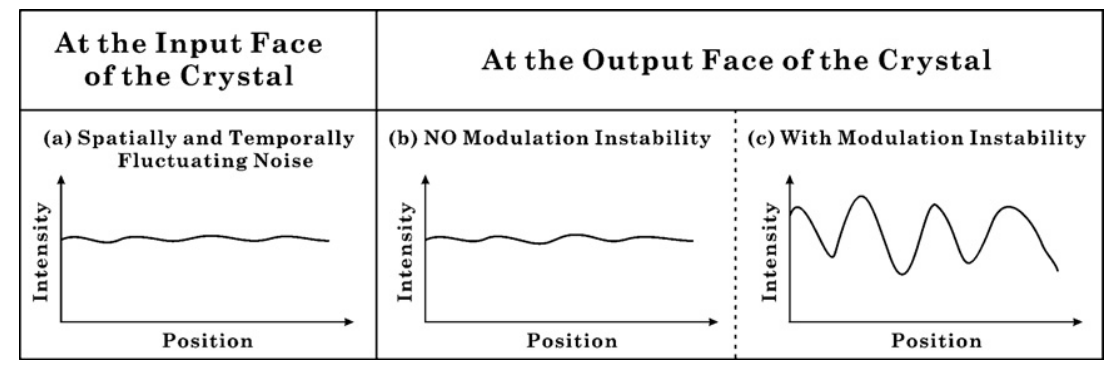

Fig. 1. Illustration of the modulation instability and pattern formation.

bicomponent solitons in dynamic soliton-like modes [7] and with the swinging solitons [8]. Motivated by the reports of the incoherent modulation instability, we are very interested again in exploring how a spatially and temporally varying optical spatial soliton beam behaves in a somewhat instantaneous nonlinear medium.

In a previous paper [9], we have ever reported another series of experiments on the pattern dynamics [10] due to the modulation instability by launching a very broad coherent $\mathrm{CW}$ light beam uniformly onto a biased photorefractive strontium barium niobate crystal (SBN:60, $n=2.35$ and $\left.r_{33}=280 \mathrm{pm} / \mathrm{V}\right)$. When the external voltage or the incident light intensity exceeds a specific threshold, we observe that the modulation instability breaks into a novel optical turbulence [11], which behaves as a quasihomogeneous speckled beam or a partially incoherent beam. This spatiotemporal chaotic behavior reveals that, with nonlinearity magnitude large enough or nonlinearity relaxation time short enough, the medium can respond not only to the spatially fluctuating light intensity, but also to the temporally fluctuating light intensity due to the noise.

In this paper, we intend to further investigate the spatial coherence property of an optical turbulent beam [12], which also behaves as a quasi-homogeneous speckled beam or a partially incoherent beam. The beam size is as broad as $200 \mu \mathrm{m}$ around, and is about 10 times larger than that of an ordinary optical spatial soliton formed in a biased photorefractive crystal. We adopt the strategy of measuring the double-slit-interference fringes [13] of the broad optical turbulent beam. The equivalent experimental methods and results could also be found in other fields of optical physics [14].

Turbulence is of large importance in many fields. In addition to the turbulent flow in ordinary fluids, the quantum turbulence was also studied in superfluid helium-4 or helium-3 at very low temperatures [15]. It implies that a turbulent flow might be not solely a classical phenomenon. How the quantum effects affect an optical turbulent beam in a biased photorefractive crystal could become a new research issue in the future. The non-classical coupling or interaction between the dynamic multi-quasi-solitons and the fast-moving soliton-induced multi-waveguides of quasi-photonic-crystal structure for an optical turbulent beam in a biased photorefractive crystal will also probably attract again many research interests.

In classical fluid dynamics, one can measure the pressure gradients associated with the turbulent flow in a tube and the forces on the obstacles which the turbulent flow passes [15]. The vortex lines exhibit the presence of viscosity or mutual frictional interaction. One can obtain the density of vortex lines or the vorticity in flows through the measurement of mutual friction. Another important measurement of the pressure fluctuations, which is related to the fluctuations in turbulent flow velocities, can be used to obtain the turbulent energy spectral distribution over different eddy sizes. These techniques or approaches will also

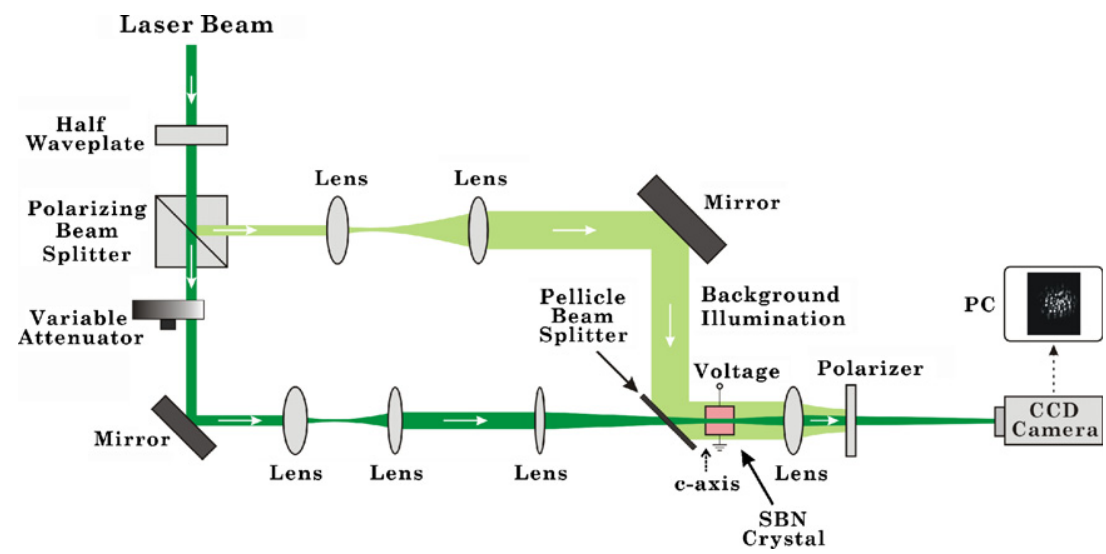

Fig. 2. The experimental setup for observing the spatiotemporal pattern formation of an optical turbulent beam. 
inspire us to pursue deeper understanding of the optical turbulent beam in a biased photorefractive crystal due to the spatiotemporal modulation instability in the future.

\section{Observing the spatiotemporal pattern formation of an optical turbulent beam}

The experimental setup for observing an optical turbulent beam is shown in Fig. 2, and is similar to the typical setup for observing a photorefractive optical spatial soliton. At first, to produce a broad turbulent beam, a $\mathrm{TEM}_{00}$ extraordinarily polarized signal laser beam (wavelength at $532 \mathrm{~nm}$ ) is focused by a lens of $1000-\mathrm{mm}$ focal length and then launched into the SBN crystal along the 7-mm-long crystalline $a$-axis, with the $195-\mu \mathrm{m}$-wide (FWHM) minimum beam waist located at the crystal input face. The direction of the crystalline $c$-axis is parallel to the polarization of the signal light beam. Thus we can take advantage of the nonlinear photorefractive effect of the crystal to produce the modulation instability. At a laser output power of $0.3 \mathrm{~W}$, the power of the incident signal beam is measured to be about $0.23 \mu \mathrm{W}$ in this system. Another ordinarily polarized beam is expanded and sent through the crystal together with the signal beam, as a uniform background beam. The intensity ratio of the signal-beam peak intensity and the averaged background illumination is 4.7 roughly. The image of the light beam at the input or the output face of the crystal is projected onto a charge-coupled device (CCD) camera by a focusing lens.

In our system, when an external DC bias voltage is applied upon the two electrodes sandwiching the 5-mmwide crystalline $c$-axis, the extraordinarily polarized light beam experiences a negative refractive index change via the electro-optic effect, given the bias polarity is opposite to the crystalline $c$-axis. In this situation, as the externally applied voltage is large enough, the bright-type noise will be amplified spontaneously by the self-focusing effect induced by the photorefractive screening nonlinearity, forming filaments pattern due to the modulation instability $[9,16,17]$. Nevertheless, it has been proved that the induced spatiotemporal modulation instability can also exist in a self-defocusing medium if the nonlinearity is noninstantaneous [18]. This is another important issue to be discussed and not considered now in this study.

Fig. 3 shows the measured images of the modulation instability patterns of the signal beam at the crystal output face at various external voltages under a fixed laser output power of $0.3 \mathrm{~W}$. The displayed pattern diagram is the intensity distribution of the light beam at the output face of the nonlinear crystal. As the external voltage is increased further, the stripes break up into arrays of light spots, displaying larger modulation instability. Nevertheless, when the external voltage is increased further across a specific threshold, the fragmented light spots become even smaller and fast fluctuate both spatially and temporally, forming an optical turbulence due to the spatiotemporal modulation

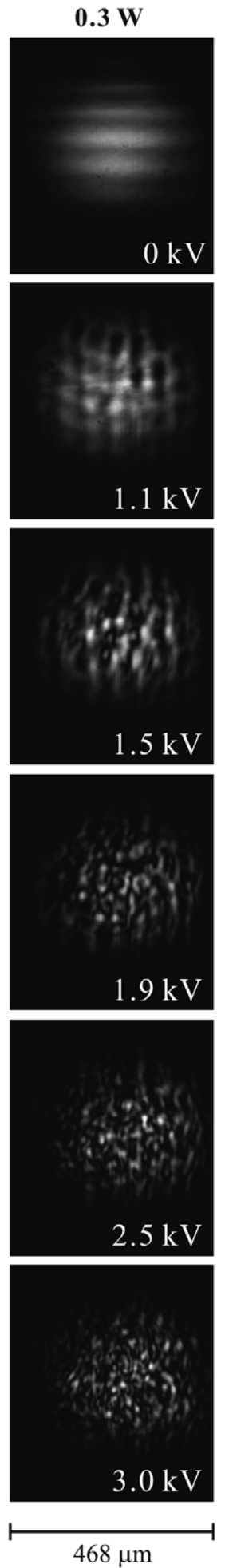

Fig. 3. Typical measured examples of the modulation instability and pattern formation of a coherent light beam in a biased photorefractive crystal at various external voltages, under a fixed laser output power of $0.3 \mathrm{~W}$.

instability, which behaves as a quasi-homogeneous speckled beam or a partially incoherent beam. We conjecture that this behavior may be related to that of the viscous flow in fluid mechanics [19]. When the optical nonlinearity (flow 
rate) reaches some critical value, the modulation instability pattern (laminar flow) undergoes a transition into the optical turbulence (turbulent flow). In Ref. [11], the spatiotemporal dynamics of patterns of the light beam emitted by a photorefractive oscillator has also been investigated in the case of very large transverse dimensions. The time and space averagings show that the transition to a turbulence as transverse dimensions are increased is a two-step process, which depends on the generalized Fresnel number, analogous to the aspect ratio in hydrodynamics. A threshold dependence on the applied nonlinearity or input conditions for a turbulence to take place is a common phenomenon.

In Fig. 3, we can also see that, in the modulation instability process, the periodicity of the filaments, or the size of the light spots, depends on the magnitude of the external voltage. For the sake of the combined effects of the nonlinear self-focusing effect and the light diffraction, the filament spatial frequency increases and the spot size decreases, with increasing nonlinearity magnitude. On the other hand, notice that, due to the crystal striations generated during the manufacturing process, the broad incident light beam is made multi-broadband shape (with separation of about $68 \mu \mathrm{m}$ ) after passing through the unbiased crystal (Fig. 3, at $0 \mathrm{kV}$ ). At the same while, the self-bending [20,21] of the broad turbulent signal beam, due to the increasingly strong diffusion field, is also observed when the broad signal beam breaks up into small chaotic light spots at higher external voltages (Fig. 3, at $3.0 \mathrm{kV}$ ).

In fluid dynamics, there is a common example, which considers a turbulent flow of water past a cylindrical obstacle. The Reynolds number, a dimensionless velocity parameter, is defined as $R e=\rho V D / \eta$, where $\rho$ is the water's density, $V$ is the free-stream's velocity, $D$ is the cylinder's diameter, and $\eta$ is the water's viscosity [15]. If the Reynolds number increases, the down-stream eddies behind the obstacle will become smaller. By analogy, we also find that, for an optical turbulent beam in a biased photorefractive crystal due to the spatiotemporal modulation instability, as the external voltage or the optical intensity increases, the fragmented speckle size will become smaller and the fluctuating speed of chaotic light spots will become faster [9].

\section{Measuring the spatial coherence of an optical turbulent beam}

Next, as shown in Fig. 4, to elucidate the spatial coherence property of the turbulent signal beam due to the modulation instability, we perform a series of measurements on the optical interference fringes of the turbulent signal beam by a double-slit plate of $40-\mu \mathrm{m}$ slit width and $250-\mu \mathrm{m}$ slit separation. This double-slit plate is placed at the original position (the image plane of the imaging lens) of the slightly withdrawn CCD camera, and corresponds to 4.6- $\mu \mathrm{m}$ slit width and $28.7-\mu \mathrm{m}$ slit separation at the input or the output face of the crystal in our imaging system. The averaged interference fringes of the turbulent signal beam for various external voltages at a fixed laser output power of $1.5 \mathrm{~W}$ are shown in Fig. 5a. The higher the external voltage, the faster the speed of the optical turbulence, and thus the lower the visibility of the averaged interference fringe (or the degree of the optical spatial coherence). The fringe visibility could be defined as $\left(I_{\max }-I_{\min }\right) /$ $\left(I_{\max }+I_{\min }\right)$, where $I_{\max }$ and $I_{\min }$ are the maximum and minimum light intensities of the central fringe [13]. Besides, the averaged interference fringes of the turbulent signal beam for various laser output powers at a fixed external voltage of $1.5 \mathrm{kV}$ are shown in Fig. 5b. At higher laser output power, the visibility of the averaged interference fringes is also reduced down, demonstrating explicitly that the input coherent light beam could also be made more incoherent by the spatiotemporal modulation instability at higher incident light intensity. This is because, at higher light intensity, the medium can respond more quickly to the spatially and temporally fluctuating light intensity due to the noise. The relationships of the visibility of the averaged double-slit interference fringe versus the external voltage or the laser output power are plotted in Fig. 6. They clearly demonstrate that the visibility of the averaged interference fringe (or the degree of the optical spatial coherence) is inversely varied with increasing external voltage of the crystal or the laser output power. The intensity profile of the averaged interference fringe is obtained by averaging all the snapshot images (50 frames) within a duration of $7.5 \mathrm{~s}$ (Fig. 5a) or $10 \mathrm{~s}$ (Fig. 5b).

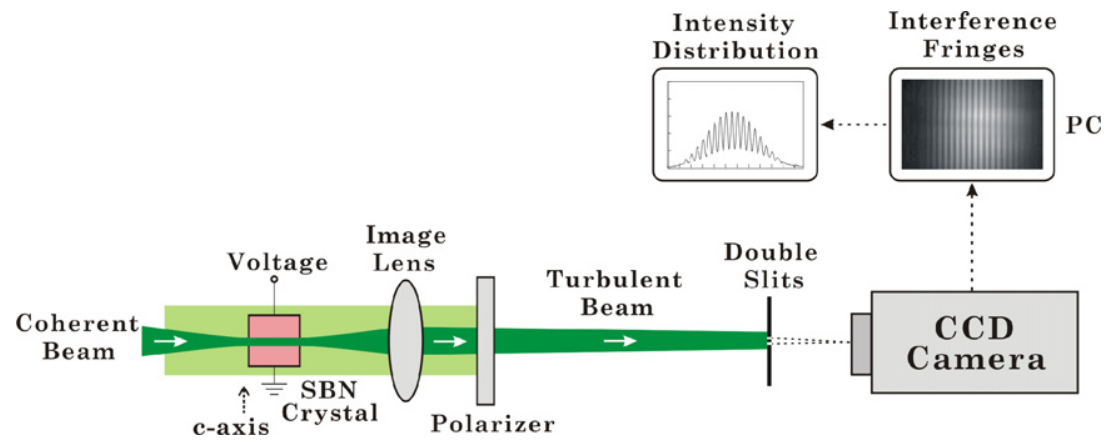

Fig. 4. The experimental setup for investigating the spatial coherence property of an optical turbulent beam using the method of double-slit interference. 

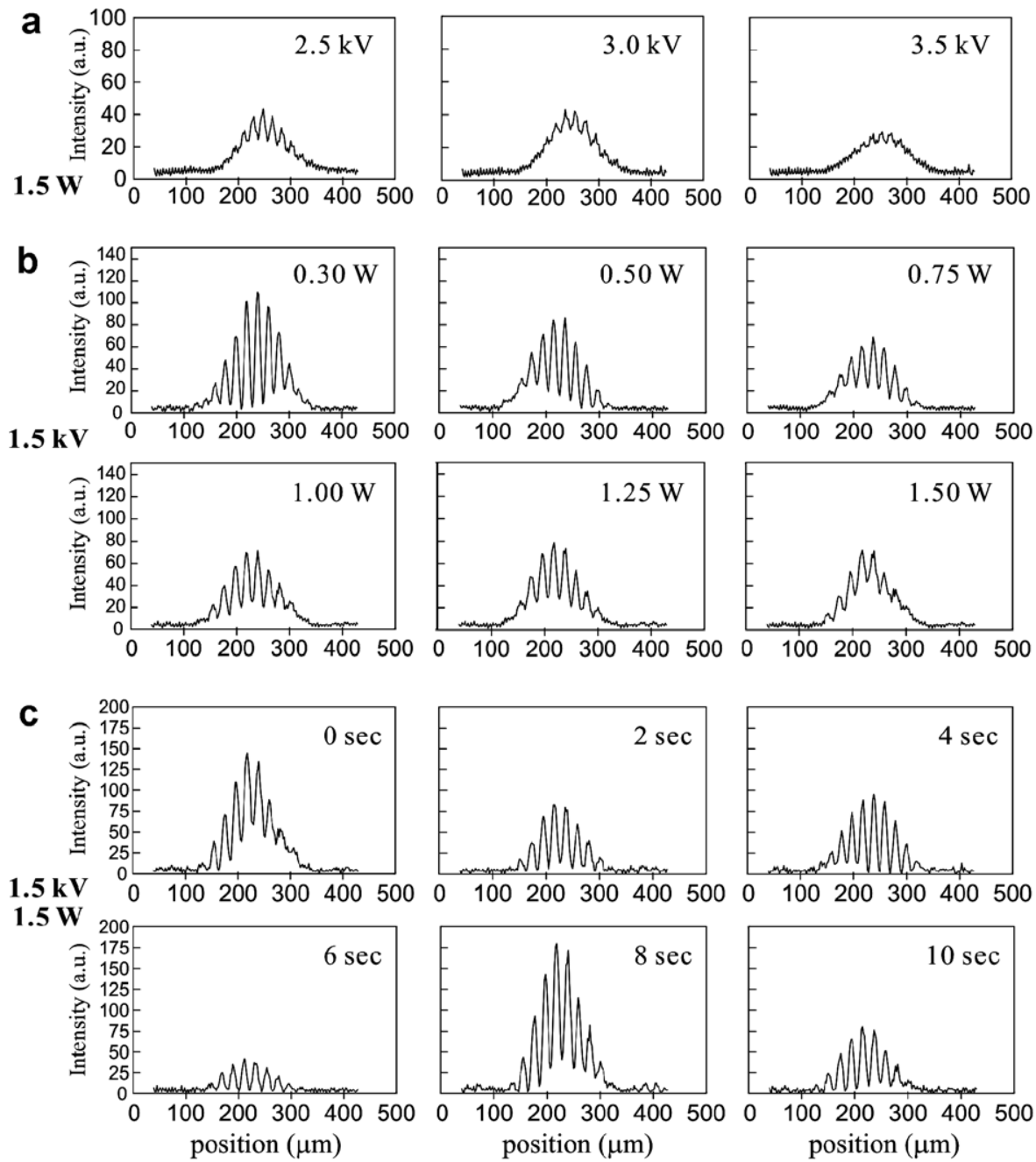

Fig. 5. The averaged double-slit interference fringes of the optical turbulent beam, (a) for various external voltages at a fixed laser output power of $1.5 \mathrm{~W}$, and (b) for various laser output powers at a fixed external voltage of $1.5 \mathrm{kV}$. (c) An example of several snapshot interference fringes for a series of specified instants, at a laser output power of $1.5 \mathrm{~W}$ and an external voltage of $1.5 \mathrm{kV}$.

Owing to the typical low optical intensity, the response of the photorefractive crystal is quite slow, and thus the fluctuating speed of the turbulent light spots is not quite fast. As a result, sometimes the double slits see "one" bigger light spot (higher spatial coherence), but some times they see "several" smaller light spots of different intensities simultaneously (lower spatial coherence). Therefore, compared to that of the averaged interference fringe (Fig. 5b, last picture), the visibility of the transient interference fringe is changing higher or lower as the turbulent beam is fluctuating. An example of several snapshot time-varying interference fringes at a series of specified instants is shown in Fig. 5c, at a laser output power of $1.5 \mathrm{~W}$ and an external voltage of $1.5 \mathrm{kV}$. As a result, we must have carried out the temporal averaging procedure to indicate the overall optical coherence characteristics of the quasi-homogeneous speckled beam (or the partially incoherent beam) due to the spatiotemporal modulation instability in a biased photorefractive crystal.
The visibility fluctuation of the instantaneous double-slit interference fringe of an optical turbulent beam tells us the fact that the fragmented coherent light spots incident on the double slits are varying spatially and temporally. Therefore, from another point of view, the visibility of the averaged interference fringe can also be related equivalently to the temporal coherence of the optical turbulent beam. To elucidate the picture of the visibility fluctuation, we perform a series of calculations about the intensity profiles of the interference fringes for chaotic incident coherent light spots by the theory of double-slit interference [13]. Suppose the slit separation (center to center) is $a$, and the slit width is $b$. The far-field diffraction pattern of a plane wavefront that is obstructed by two narrow slits, on which the field amplitudes are different, can be found approximately by integrating the total electric field at a point $\mathrm{P}$ on the screen, over the widths of the two slits, respectively. So we find, 

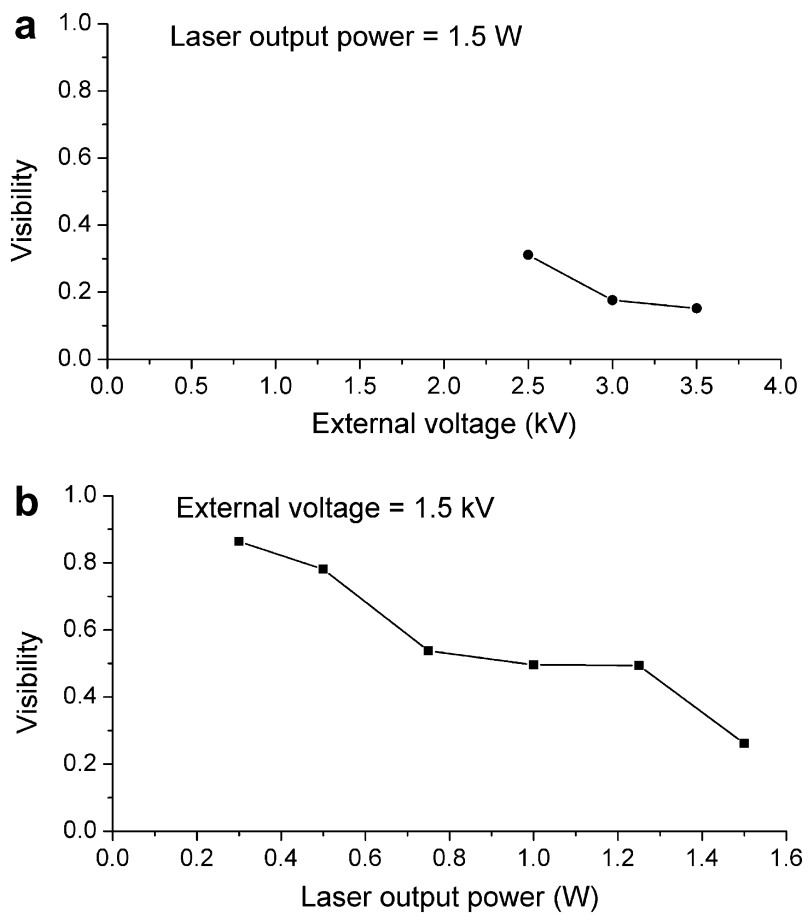

Fig. 6. The visibilities of the averaged double-slit interference fringes of the optical turbulent beam, (a) for various external voltages at a fixed laser output power of $1.5 \mathrm{~W}$, and (b) for various laser output powers at a fixed external voltage of $1.5 \mathrm{kV}$.

$$
\begin{aligned}
E_{\mathrm{p}}= & \int_{(a-b) / 2}^{(a+b) / 2} \frac{E_{1}}{r_{0}} \mathrm{e}^{\mathrm{i}\left(k r_{0}-\omega t\right)} \mathrm{e}^{\mathrm{i} k s \sin \theta} \mathrm{d} s \\
& +\int_{(-a-b) / 2}^{(-a+b) / 2} \frac{E_{2}}{r_{0}} \mathrm{e}^{\mathrm{i}\left(k r_{0}-\omega t\right)} \mathrm{e}^{\mathrm{i} k s \sin \theta} \mathrm{d} s \\
= & \frac{E_{1}}{r_{0}} \mathrm{e}^{\mathrm{i}\left(k r_{0}-\omega t\right)}\left(\frac{\mathrm{e}^{\mathrm{i} k s \sin \theta}}{\mathrm{i} k \sin \theta}\right)_{(a-b) / 2}^{(a+b) / 2}+\frac{E_{2}}{r_{0}} \mathrm{e}^{\mathrm{i}\left(k r_{0}-\omega t\right)}\left(\frac{\mathrm{e}^{\mathrm{i} k s \sin \theta}}{\mathrm{i} k \sin \theta}\right)_{(-a-b) / 2}^{(-a+b) / 2} \\
= & \frac{E_{1}}{r_{0}} \mathrm{e}^{\mathrm{i}\left(k r_{0}-\omega t\right)} \frac{\left[\mathrm{e}^{\mathrm{i} k[(a+b) / 2] \sin \theta}-\mathrm{e}^{\mathrm{i} k[(a-b) / 2] \sin \theta}\right]}{\mathrm{i} k \sin \theta} \\
& +\frac{E_{2}}{r_{0}} \mathrm{e}^{\mathrm{i}\left(k r_{0}-\omega t\right)} \frac{\left[\mathrm{e}^{\mathrm{i} k[(-a+b) / 2] \sin \theta}-\mathrm{e}^{\mathrm{i} k[(-a-b) / 2] \sin \theta]}\right.}{\mathrm{i} k \sin \theta},
\end{aligned}
$$

where $E_{1}$ and $E_{2}$ are the amplitude proportionality constants of the electric fields, $k$ is the propagation constant, $\omega$ is the angular frequency, $r_{0}$ is the mean optical-path length from the slit to the screen, $t$ is the time, $\theta$ is the angle of the optical-path under observation to the normal line, and $s$ is the spatial coordinate along the slits. Using the convenient substitutions,

$\alpha=k(a / 2) \sin \theta, \quad$ and $\quad \beta=k(b / 2) \sin \theta$,

the integration above gives

$$
\begin{aligned}
E_{\mathrm{p}}= & \frac{E_{1}}{r_{0}} \mathrm{e}^{\mathrm{i}\left(k r_{0}-\omega t\right)} \frac{b / 2}{i \beta}\left[\mathrm{e}^{\mathrm{i}(\alpha+\beta)}-\mathrm{e}^{\mathrm{i}(\alpha-\beta)}\right]+\frac{E_{2}}{r_{0}} \mathrm{e}^{\mathrm{i}\left(k r_{0}-\omega t\right)} \\
& \times \frac{b / 2}{i \beta}\left[\mathrm{e}^{\mathrm{i}(-\alpha+\beta)}-\mathrm{e}^{\mathrm{i}(-\alpha-\beta)}\right] \\
= & \frac{E_{1}}{r_{0}} \mathrm{e}^{\mathrm{i}\left(k r_{0}-\omega t\right)} \frac{b / 2}{i \beta}\left[\mathrm{e}^{\mathrm{i} \alpha}\left(\mathrm{e}^{\mathrm{i} \beta}-\mathrm{e}^{-\mathrm{i} \beta}\right)\right]+\frac{E_{2}}{r_{0}} \mathrm{e}^{\mathrm{i}\left(k r_{0}-\omega t\right)} \\
& \times \frac{b / 2}{i \beta}\left[\mathrm{e}^{-\mathrm{i} \alpha}\left(\mathrm{e}^{\mathrm{i} \beta}-\mathrm{e}^{-\mathrm{i} \beta}\right)\right] \\
= & \frac{1}{r_{0}} \mathrm{e}^{\mathrm{i}\left(k r_{0}-\omega t\right)} \frac{b / 2}{i \beta}\left(\mathrm{e}^{\mathrm{i} \beta}-\mathrm{e}^{-\mathrm{i} \beta}\right)\left(E_{1} \mathrm{e}^{\mathrm{i} \alpha}+E_{2} \mathrm{e}^{-\mathrm{i} \alpha}\right) \\
= & \frac{b}{r_{0}} \mathrm{e}^{\mathrm{i}\left(k r_{0}-\omega t\right)} \frac{\sin \beta}{\beta}\left(E_{1} \mathrm{e}^{\mathrm{i} \alpha}+E_{2} \mathrm{e}^{-\mathrm{i} \alpha}\right) .
\end{aligned}
$$

Thus, we derive the optical intensity of the diffraction pattern to be

$I=\frac{\varepsilon_{0} c}{2}\left|E_{\mathrm{p}}\right|^{2}=\frac{\varepsilon_{0} c}{2}\left(\frac{b}{r_{0}}\right)^{2}\left(\frac{\sin \beta}{\beta}\right)^{2}\left|E_{1} \mathrm{e}^{\mathrm{i} \alpha}+E_{2} \mathrm{e}^{-\mathrm{i} \alpha}\right|^{2}$.

The parameter $\beta$ can be related to the parameter $\alpha$ by $\beta=(b / a) \alpha$. We plot the intensity distributions of the diffraction patterns for various incident field amplitudes as a function of the parameter $\alpha$ in Fig. 7, where $b / a=250 /$ $40=6.25$ and $\alpha$ is proportional to the displacement from the point observed to the fringe center on the screen. From the simulation results, we are convinced that the double-slit diffraction pattern is equivalent to the interference fringe within a single-slit diffraction envelope. We also find that the fringe visibility will vary as the amplitude ratio of the electric fields on the two slits changes. It proves that an
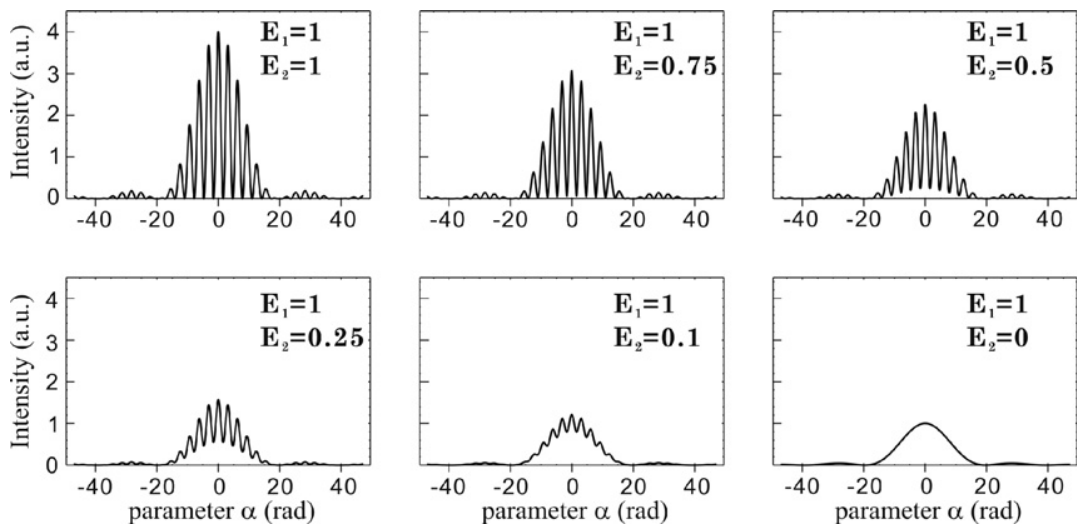

Fig. 7. The calculated intensity distributions of the double-slit diffraction patterns on the screen for various incident field amplitudes on the two slits. 
optical turbulent beam in a biased photorefractive crystal due to the spatiotemporal modulation instability is composed of chaotic coherent light spots varying spatially and temporally.

\section{Conclusion}

In conclusion, we have experimentally explored the spatiotemporal modulation instability and the pattern formation dynamics by the use of a broad coherent light beam incident in a biased photorefractive crystal. As the external voltage or the incident light intensity exceeds a threshold, the light beam forms filaments spontaneously from noise, and further breaks up into arrays of light spots at higher voltage or incident light intensity. When the external voltage or the incident light intensity is increased further across a specific threshold, the light spots become even smaller and fast fluctuate both spatially and temporally, forming an optical turbulence, which behaves as a quasihomogeneous speckled beam or a partially incoherent beam. The spatial coherence property of an optical turbulent beam is also investigated experimentally using the method of double-slit interference. The higher the external voltage or the incident light intensity, the faster the speed of the optical turbulence, and thus the lower the visibility of the averaged interference fringe (or the degree of the optical spatial coherence). The visibility variation of the instantaneous double-slit interference fringe of an optical turbulent beam is also demonstrated by a numerical simulation. More theoretical investigation and experimental work are needed to clarify the formation mechanism and model of the transition to an optical turbulence due to the spatiotemporal modulation instability in photorefractive media. These studies will help us understand more deeply about the issue of the soliton instability owing to the noninstantaneous nature of the photorefractive screening nonlinearity.

\section{Acknowledgement}

We acknowledge the financial support from the National Science Council, Taiwan, through Projects NSC89-2112-M-002-060 and NSC-95-2112-M-415-004.

\section{References}

[1] D. Kip, M. Soljacic, M. Segev, E.D. Eugenieva, D.N. Christodoulides, Science 290 (2000) 495.

[2] T.H. Coskun, D.N. Christodoulides, Y.-R. Kim, Z. Chen, M. Soljacic, M. Segev, Phys. Rev. Lett. 84 (2000) 2374.

[3] J. Klinger, H. Martin, Z. Chen, Opt. Lett. 26 (2001) 271.

[4] M. Mitchell, Z. Chen, M.-F. Shih, M. Segev, Phys. Rev. Lett. 77 (1996) 490.

[5] Z. Chen, M. Mitchell, M. Segev, T.H. Coskun, D.N. Christodoulides, Science 280 (1998) 889.

[6] M. Mitchell, M. Segev, Nature 387 (1997) 880.

[7] M.-F. Shih, F.-W. Sheu, Phys. Rev. Lett. 86 (2001) 2281.

[8] F.-W. Sheu, M.-F. Shih, J. Opt. A: Pure Appl. Opt. 9 (2007) 271.

[9] M.-F. Shih, C.-C. Jeng, F.-W. Sheu, C.-Y. Lin, Phys. Rev. Lett. 88 (2002) 133902.

[10] A.V. Mamaev, M. Saffman, D.Z. Anderson, A.A. Zozulya, Phys. Rev. A 54 (1996) 870.

[11] J. Farjas, D. Hennequin, D. Dangoisse, P. Glorieux, Phys. Rev. A 57 (1998) 580.

[12] L.C. Andrews, W.B. Miller, J.C. Ricklin, J. Opt. Soc. Am. A 11 (1994) 1653.

[13] F.L. Pedrotti, L.M. Pedrotti, L.S. Pedrotti, Introduction to Optics, third ed., Prentice Hall, 2006 (Chapter 9 and 11).

[14] N.K.M.N. Srinivas, S.S. Harsha, D.N. Rao, Opt. Express 13 (2005) 3224.

[15] W.F. Vinen, R.J. Donnelly, Phys. Today 60 (2007) 43.

[16] M. Saffman, G. McCarthy, W. Królikowski, J. Opt. B: Quant. Semiclass. Opt. 6 (2004) S397.

[17] H. Wang, W. She, Chinese Opt. Lett. 4 (2006) 108.

[18] W.-H. Chu, C.-C. Jeng, C.-H. Chen, Y.-H. Liu, M.-F. Shih, Opt. Lett. 30 (2005) 1846.

[19] L.D. Landau, E.M. Lifshitz, Fluid Mechanics, third ed., ButterworthHeinemann, Oxford, 1997 (Chapter 2).

[20] M.-F. Shih, P. Leach, M. Segev, G.C. Valley, G. Salamo, B. Crosignani, P. DiPorto, M. Garret, Opt. Lett. 21 (1996) 324.

[21] S.R. Singh, D.N. Christodoulides, Opt. Commun. 118 (1995) 569. 